\title{
DAMN: A Distributed Architecture for Mobile Navigation
}

\author{
Julio K. Rosenblatt \\ Robotics Institute \\ Carnegie Mellon University \\ Pittsburgh, PA 15213 \\ jkr@cmu.edu
}

\begin{abstract}
An architecture is presented in which distributed taskachieving modules, or behaviors, cooperatively determine a mobile robot's path by voting for each of various possible actions. An arbiter then performs command fusion and selects that action which best satisfies the prioritized goals of the system, as expressed by these votes, without the need to average commands. Command fusion allows multiple goals and constraints to be considered simultaneously. Examples of implemented systems are given, and future research directions in command fusion are discussed.
\end{abstract}

Keywords: mobile robots, distributed architecture, behaviors, voting, arbitration, command fusion

\section{Introduction}

In order to function in unstructured, unknown, or dynamic environments, a mobile robot must be able to perceive its surroundings and generate actions that are appropriate for that environment and for the goals of the robotic system. Mobile robots need to combine information from several different sources. For example, the CMU Navlab vehicles are equipped with sensors such as video cameras, laser range finders, sonars, and inertial navigation systems, which are variously used by subsystems that follow roads, track paths, avoid obstacles and rough terrain, seek goals, and perform teleoperation. Because the raw sensor data and the internal representations used by these subsystems tend to vary widely, especially when these modules have been developed independently, combining them into one coherent system which combines all their capabilities has proven to be very difficult.

To function effectively, an architectural framework for these sensing and reasoning processes must be imposed to provide a structure with which the system may be developed, tested, debugged, and understood. However, the architecture should serve as an aid, not a burden, in the integration of modules that have been developed independently, so it must not be overly restrictive. It must allow for purposeful goaloriented behavior yet retain the ability to respond to potentially dangerous situations in real-time while maintaining enough speed to be useful.

The earliest work in robot control architectures attempted to reason by manipulating abstract symbols using only pure logic (Nilsson, 1984). The limitations of this top-down AI approach led to a new generation of architectures designed in a bottom-up fashion to provide greater reactivity to the robot's surroundings, but sacrificed generality and the ability to reason about the system's own intentions and goals.

Hierarchical approaches allow slower abstract reasoning at the higher levels and faster numerical computations at the lower levels, thus allowing varying trade-offs between responsiveness and optimality as appropriate at each level (Payton, 1986; Albus, McCain and Lumia, 1987). While such an approach provides aspects of both deliberative planning and reactive control, the top-down nature of hierarchical structures tends to overly restrict the lower levels (Payton, Rosenblatt and Keirsey, 1990). In hierarchical architectures, each layer controls the layer beneath it and assumes that its commands will be executed as expected. Since expectations are not always met, there is a need to monitor the progress of desired actions and to report failures as they occur (Simmons, Lin and Fedor, 1990). In an unstructured, unknown, or dynamic environment, this approach introduces complexities and inefficiencies which could be avoided if higher level modules participated in the decision-making process without assuming that their commands will be strictly followed.

Rather than imposing a top-down structure to achieve this desired symbiosis of deliberative and reactive elements, the Distributed Architecture for Mobile Navigation takes an approach where multiple modules concurrently share control of the robot by sending votes to be combined rather than commands to be selected and executed (Rosenblatt, 1995; Rosenblatt and Thorpe, 1995).

The Distributed Architecture for Mobile Navigation has been successfully used to integrate the various subsystems mentioned above, thus providing systems that perform tasks such as road following, cross-country navigation, and teleoperation while avoiding obstacles and meeting mission objectives. In addition to its use on the CMU Navlab vehicles, DAMN has also been used at Martin Marietta, Hughes Research Labs, and the Georgia Institute of Technology.

\section{The Distributed Architecture for Mobile Navigation}

Deliberative planning and reactive control are equally important for mobile robot navigation; when used appropriately, each complements the other and compensates for the other's deficiencies. Reactive components provide the basic capabilities which enable the robot to achieve lowlevel tasks without injury to itself or its environment, while 
deliberative components provide the ability to achieve higher-level goals and to avoid mistakes which could lead to inefficiencies or even mission failure. But rather than imposing an hierarchical structure to achieve this symbiosis, the Distributed Architecture for Mobile Navigation (DAMN) takes an approach where multiple modules concurrently share control of the robot. In order to achieve this, a scheme is used where each module votes for or against various alternatives in the command space based on geometric reasoning, without regard for the level of planning involved.

Figure 1 shows the organization of the DAMN architecture, in which individual behaviors such as road following or obstacle avoidance send votes to the command arbitration module; these inputs are combined and the resulting command is sent to the vehicle controller. Each action-producing module, or behavior, is responsible for a particular aspect of vehicle control or for achieving some particular task; it operates asynchronously and in parallel with other behaviors, sending its outputs to the arbiter at whatever rate is appropriate for that particular function. Each behavior is assigned a weight reflecting its relative priority in controlling the vehicle. A mode manager may also be used to vary these weights during the course of a mission based on knowledge of which behaviors would be most relevant and reliable in a given situation.

DAMN is a behavior-based architecture similar in some regards to reactive systems such as the Subsumption Architecture (Brooks, 1986). In contrast to more traditional centralized AI planners that build a centralized world model and plan an optimal path through it, a behavior-based architecture consists of specialized task-achieving modules that operate independently and are responsible for only a very narrow portion of vehicle control, thus avoiding the need for sensor fusion. A distributed architecture has several advantages over a centralized one, including greater reactivity, flexibility, and robustness (Payton, Rosenblatt and Keirsey, 1990). However, one important distinction between this system and purely reactive systems is that, while an attempt is made to keep the perception and planning components of a behavior as simple as possible without sacrificing dependability, they can and often do maintain internal representations of the world. Brooks (1993) has argued that "the world is its own best model", but this assumes that the vehicle's sensors and the algorithms which process them can not benefit from evidence combination between consecutive scenes. In addition, disallowing the use of internal representations requires that all environmental features of immediate interest be visible to the vehicle sensors at all times; thus adding unnecessary constraints and reducing the flexibility of the overall vehicle system

The DAMN architecture is designed to provide the basic capabilities essential to any mobile robot system, or first level of competence in the parlance of the Subsumption Architecture. In DAMN, this consists of safety behaviors which limit turn and speed to avoid vehicle tip-over or wheel slippage, obstacle avoidance behaviors to prevent collisions, as well as various auxiliary behaviors (see DAMN Behaviors section). As new functions are needed, additional behaviors can be added to the system without any need for modification to the previously included behaviors, thus preserving their established functionality.

Since both deliberative and reflexive modules are needed, DAMN is designed so that behaviors can issue votes at any rate; for example, one behavior may operate reflexively at $10 \mathrm{~Hz}$, another may maintain some local information and operate at $1 \mathrm{~Hz}$, while yet another module may plan optimal paths in a global map and issue votes at a rate of $0.1 \mathrm{~Hz}$. The use of distributed shared control allows multiple levels of planning to be used in decisionmaking without the need for an hierarchical structure. However, higher-level reasoning modules may still exert meta-level control within DAMN by modifying the voting weights assigned to behaviors and thus controlling the degree to which each behavior may influence the system's decision-making process and thus the robot's actions.

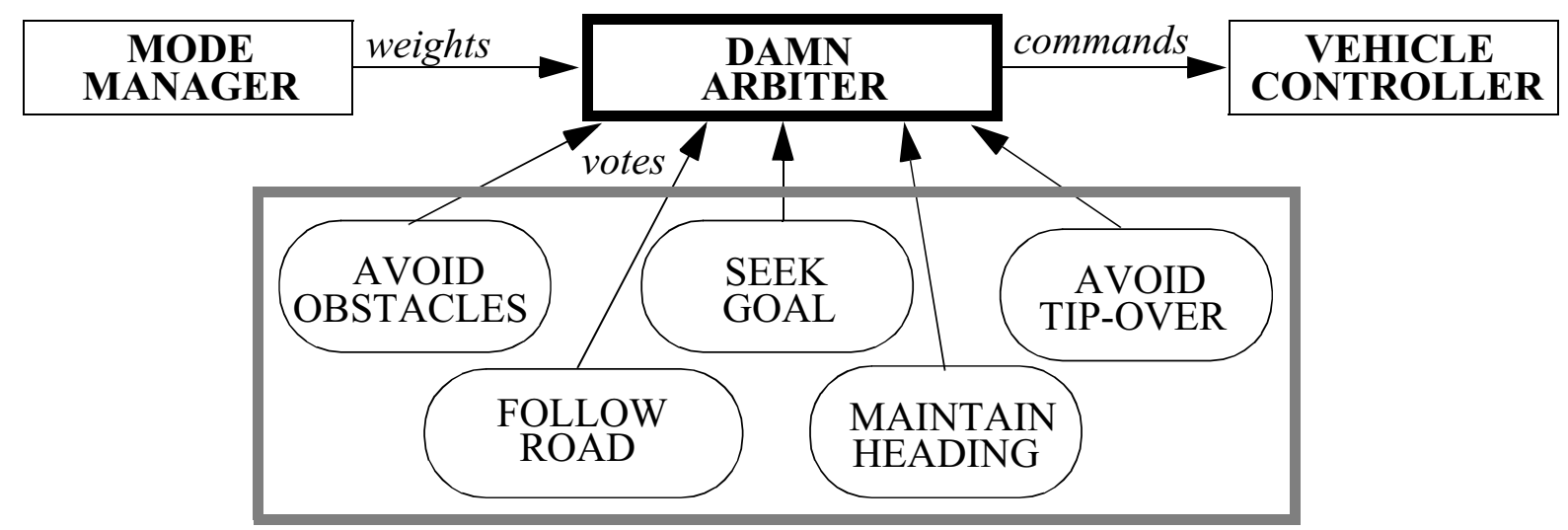

Figure 1: Overall structure of DAMN. 


\section{Command Fusion}

Centralized, blackboard, and hierarchical architectures perform sensor fusion in order to construct a coherent world model which is then used for planning actions. This approach has the advantage of being able to combine data from previous world models to overcome ambiguities and noise inherent in the sensing process, but has the disadvantage of creating a computationally expensive sensory bottleneck - all sensor data must be collected and integrated before it can be acted upon. Furthermore, information from disparate sources such as maps, sonar, and video, are generally not amenable to combination within a single representational framework that is suitable for planning such dissimilar tasks as following roads and avoiding obstacles, and a single monolithic world model is also more difficult to develop, maintain, and extend.

In contrast, behavior-based architectures do not need to create a central world model; instead, the perceptual processing is distributed across multiple independent modules. Each behavior requires only fragmentary knowledge of the world and receives exclusively that sensory data which is directly relevant to its particular decision-making needs, so there is no need to fuse all available data into a single coherent world model. Based on this input, each behavior then produces a desired action, and the system must use these independently produced outputs to control the robot.

By appropriately fusing behavior commands through arbitration, a robot control system can respond to its environment without suffering the problems inherent in sensor fusion. Instead of performing sensor fusion, the system must combine command inputs to determine an appropriate course of action.

\section{Priority-Based Command Selection}

In a distributed architecture, it is necessary to decide which behaviors should be controlling the vehicle at any given time. In some architectures, this is achieved by having priorities assigned to each behavior; of all the behaviors issuing commands, the one with the highest priority is in control and the rest are ignored. In the Subsumption Architecture (Brooks, 1986), this prioritization is implicit in the wiring of behavior modules; a higher-level behavior can override the output of a lower-level behavior via an inhibition link. In the Gapps architecture (Rosenschein and Kaelbling, 1986), such priorities are compiled into a mediator which combines inputs from the behaviors through a combinatorial logic circuit which yields the system output; as in the Subsumption Architecture, behaviors consist of finite state automata. The lowest level of the hierarchical architecture developed at the Hughes Research Labs also consists of a similar distributed control mechanism; a centralized blackboard is used for arbitration and communications purposes; priorities are explicit and can be changed dynamically (Payton, 1986). In (Maes, 1991), behavior selection is achieved via activation and inhibition links in what is effectively a winner-take-all network.

In these architectures, the satisfaction of multiple, possibly conflicting goals is achieved by having one behavior's commands completely override another's. While this is an effective scheme for choosing among incompatible commands, it does not provide an adequate means for dealing with multiple goals that can and should be satisfied simultaneously. This inability to compromise stems from the fact that priority-based arbitration of commands masks all of the knowledge within each individual behavior that was used to reach its decision. In the process of selecting a command, behaviors may weigh several alternatives before making its ultimate choice. In other behaviors, it may be more natural to merely rule out certain inappropriate actions rather than selecting a desirable one, yet their only means of expression is to completely suppress or inhibit the outputs of other behaviors. A compromise between behaviors cannot be achieved in such an all-or-nothing scenario; whenever one behavior's output is inhibited by another, the information and knowledge represented by that behavior is completely lost to the system (Rosenblatt and Payton, 1989).

\section{DAMN Arbiters}

In order to allow multiple considerations to affect vehicle actions concurrently, DAMN uses a scheme where each behavior votes for or against each of a set of possible vehicle actions (Rosenblatt and Payton, 1989). An arbiter then performs command fusion to select the most appropriate action. Although all votes must pass through the command arbiter before an action is taken, the function provided by the arbiter is fairly simple and does not represent the centralized bottleneck of more traditional systems. While the Motor Schema framework (Arkin, 1987) also offers a means of fusing commands from multiple behaviors, it suffers from the well known problem of local minima in potential fields. Another, perhaps more serious problem, is that arbitration via vector addition can result in a command which is not satisfactory to any of the contributing behaviors. DAMN arbiters do not average commands, but rather select the command which has the most votes from the behaviors.

\section{Turn Arbiter}

In the case of the turn arbiter, each behavior generates a vote between -1 and +1 for every possible steering command, with negative votes being against and positive votes for a particular command option. The arbiter collects the new votes from each behavior that has sent them, and performs a normalized weighted sum to find the turn command with the maximum vote value. In order to avoid problems with discretization such as biasing and "bang-bang control" (i.e., alternating between discrete values in order to achieve an intermediate value), the arbiter performs sub-pixel 
interpolation. This is done by first convolving the votes with a Gaussian mask to smooth the values and then selecting the command option with the highest resulting value. A parabola is then fit to that value and the ones on either side, and the peak of the parabola is used as the command to be issued to the controller. This is similar to defuzzification in Fuzzy Logic systems (Zadeh, 1973; Lee, 1990; Kamada, Naoi and Goto, 1990); indeed an architecture has been implemented which recasts DAMN into a Fuzzy Logic framework (Yen and Pfluger, 1992).

The arbitration process used in DAMN is illustrated in Figure 2, where: (a and b) the votes from behaviors are received, (c) a weighted sum of those votes is computed, nd (d), the summed votes are smoothed and interpolated to produce the resulting command sent to the vehicle controller.

a) Behavior 1, weight $=0.8$, desired curvature $=0.04$

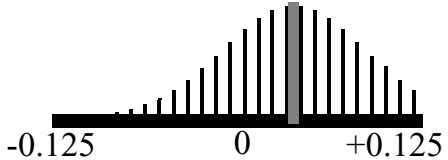

b) Behavior 2, weight $=0.2$, desired curvature $=0.0$

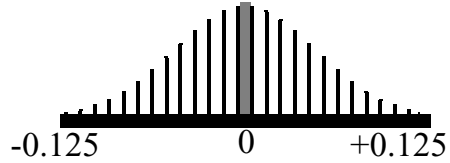

c) Weighted Sum, max vote curvature $=0.035$

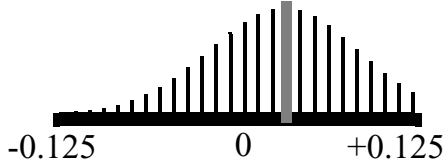

d) Smoothed and Interpolated, peak curvature $=0.033$

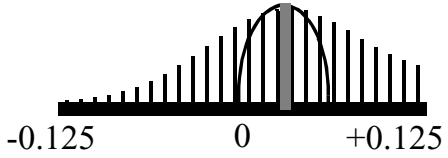

Figure 2: Command fusion process

\section{Speed Arbiter}

The emphasis in the research thus far has been in command fusion for the control of vehicle steering; until recently the commanded speed was decided in a very simplistic fashion based upon the commanded turn radius. The user-specified maximum vehicle speed was multiplied by the normalized weighted sum for the chosen turn radius; the result was the speed command issued. This is similar to the method used in Motor Schemas where the magnitude of the resultant vector determines the speed of the vehicle. Both methods suffer from the coupling of the importance that the vehicle head in a certain direction to the speed that the vehicle travels in that direction; these are independent in general.

An entirely separate DAMN speed arbiter with its own set of associated behaviors has now been developed. Thus, the turn behaviors can vote for turn commands without concern that the absolute magnitude of their votes will affect vehicle speed. At present each speed behavior votes for the largest speed possible which meets that behavior's constraints, and the arbiter simply chooses the minimum of those maxima, so that all speed constraints are satisfied.

\section{Field of Regard Arbiter}

A field of regard arbiter and its associated behaviors have also been recently implemented and used for the control of a pair of stereo cameras on a pan/tilt platform. Behaviors vote for different possible field of regard polygons (the camera field of view mapped on to the ground plane); the arbiter maintains a local map of these votes and transforms them as the vehicle moves. At each iteration, these votes are mapped into a pan-tilt space, and then smoothed and interpolated as described above. Figure 3 shows the votes for field of regard polygons generated by an behavior which votes against looking in the direction of known obstacles since travelling in that direction is impossible. Behaviors also vote based on considerations such as looking toward the goal and looking at a region contiguous to already mapped areas. The darkest polygon in the figure corresponds to the pan and tilt angles selected by the arbiter.

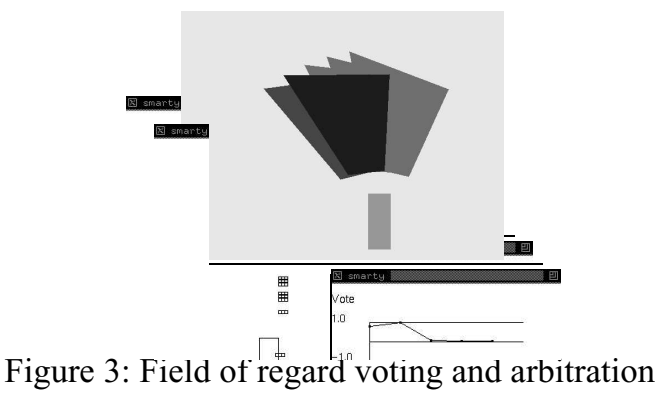

\section{DAMN Behaviors}

Within the framework of DAMN, behaviors must be defined to provide the task-specific knowledge for controlling the vehicle. Each behavior runs completely independently and asynchronously, providing votes to the arbiter each at its own rate and according to its own time constraints. The arbiter periodically sums all the latest votes from each behavior and issues commands to the vehicle controller.

\section{Safety Behaviors}

A basic need for any mobile robot system is the ability to avoid situations hazardous to itself or to other objects in its environment. Therefore, an important part of DAMN is its 
"first level of competence" (Brooks, 1986), which consists of behaviors designed to provide vehicle safety. In contrast to priority-based architectures which only allow one behavior to be effective at any given moment, the structure of DAMN and its arbitration scheme allow the function of these safety behaviors to be preserved as additional levels of competence are added.

Obstacle Avoidance The most important behavior in the context of vehicle safety is the Obstacle Avoidance behavior. In order to decide in which directions the vehicle may safely travel, this behavior receives a list of current obstacles in vehicle-centered coordinates and evaluates each of the possible command options, as illustrated in Figure 4. The source of these obstacles may be intraversable regions of terrain determined by range image processing or stereo vision, by sonar detection of objects above the ground plane, or any other means of obstacle detection as appropriate to the current task and environment (Daily et al, 1986; Langer, Rosenblatt and Hebert, 1994).

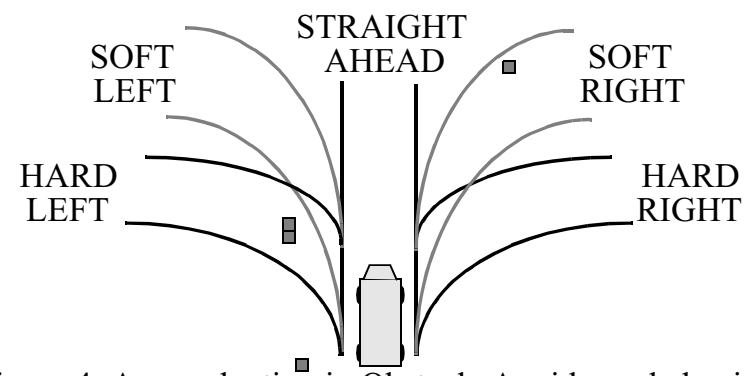

Figure 4: Arc evaluation in Obstacle Avoidance behavior

If a trajectory is completely free of any obstacles, such as the Straight Ahead or Hard Right turns shown in Figure 4, then the obstacle avoidance behavior votes for travelling along that arc. If an obstacle lies in the path of a trajectory, the behavior votes against that arc, with the magnitude of the penalty proportional to the distance from the obstacle. Thus, the Obstacle Avoidance behavior votes more strongly against those turns that would result in an immediate impact (Hard Left in the figure) and votes less strongly against those turns which would only result in a collision after travelling several meters (Soft Right). In order to avoid bringing the vehicle unnecessarily close to an obstacle, the behavior also votes against those arcs that result in a near miss (Soft Left), although the evaluation is not as unfavorable as for those trajectories leading to a direct collision.

Vehicle Dynamics Another vital aspect of vehicle safety is insuring that the commanded speed and turn stay within the dynamic constraints of the vehicle as it travels over varying terrain conditions. The most important of these constraints is the one that insures that the vehicle will not tip over. Given a velocity of magnitude $v$, the maximum positive and negative curvatures $\kappa$ to avoid tip-over would be:

$$
\pm \kappa_{\max }=\frac{ \pm(\eta \cdot g \cdot \cos \rho)+g \cdot \sin \rho}{v^{2}}
$$

where $\eta$ is the ratio of the distance between the vehicle's center of gravity (c.g.) and the wheels to the c.g. height, $g$ is the acceleration due to gravity, and $\rho$ is the vehicle roll with respect to the gravity vector. Likewise, for a given vehicle turn curvature, the maximum velocity is:

$$
v_{\text {max }}=\operatorname{Min}\left|\frac{ \pm(\eta \cdot g \cdot \cos \rho)+g \cdot \sin \rho}{\kappa}\right|^{1 / 2}
$$

Similar constraints can be imposed on vehicle turn radius and speed in order to avoid tire slippage. The limit on curvature for slippage is:

$$
\pm \kappa_{\text {max }}=\frac{(\mu \cdot g \cdot \cos \rho) \pm(g \cdot \sin \rho)}{v^{2}}
$$

where $\mu$ is the dynamic coefficient of friction between the tire and the terrain, and for speed:

$$
\pm v_{\text {max }}=\left|\frac{(\mu \cdot g \cdot \cos \rho) \pm(g \cdot \sin \rho)}{\kappa}\right|^{1 / 2}
$$

Two behaviors, Limit Turn and Limit Speed send votes to the arbiter that implement these constraints, voting against commands that violate them.

\section{Road Following}

Once vehicle safety has been assured by the obstacle avoidance and dynamic constraint behaviors, it is desirable to add additional behaviors that provide the system with the ability to achieve the tasks for which it is intended, such as road following; one of the behaviors that have been implemented within DAMN to provide this function is ALVINN.

The ALVINN road following system is an artificial neural network that is trained, using backpropagation, to associate preprocessed low resolution input images with the appropriate output steering commands (Pomerleau, 1992). In the case of ALVINN, creating a behavior that independently evaluated each arc was relatively straightforward. The units of the neural network's output layer each represent an evaluation of a particular turn command, with the layer trained to produce Gaussian curves centered about those turns that would follow the road ahead. These units are simply resampled to the DAMN voting command space, using a Gaussian of the appropriate width. This process is illustrated in Figure 5.

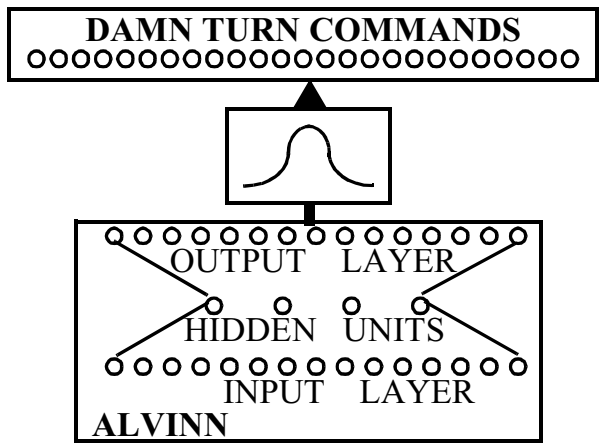

Figure 5: Resampling of ALVINN output layer 


\section{Goal-Directed Behaviors}

Another important level of functionality that should be present in any general purpose robotic system is the ability to reach certain destinations using whatever global information is available. While the low-level behaviors operate at a high rate to ensure safety and to provide functions such as road following and cross-country navigation, high-level behaviors are free to process map-based or symbolic information at a slower rate, and periodically issue votes to the arbiter that guide the robot towards the current goal.

Subgoals The Goal Seeking behavior is one way to provide this capability. This simple behavior directs the vehicle toward a series of goal points specified in global coordinates either by the user (Langer, Rosenblatt and Hebert, 1994) or by a map-based planner (Keirsey, Payton and Rosenblatt, 1988). The desired turn radius is transformed into a series of votes by applying a Gaussian whose peak is at the desired turn radius and which tapers off as the difference between this turn radius and a prospective turn command increases. A goal is considered satisfied once the vehicle enters a circle centered at the goal location; then the next goal is pursued. Because of errors in goal placement and accumulated errors in vehicle positioning, a goal point may not be reachable. For this reason, an ellipse is defined with the current goal and the subsequent goal as foci; if the vehicle enters this ellipse, the current goal is abandoned and the next one becomes the current goal instead, thus allowing progress to continue.

Dynamic Programming Some more sophisticated mapbased planning techniques have also been integrated and used within the DAMN framework. These planners use dynamic programming techniques based on the $A^{*}$ search algorithm (Nilsson, 1980) to determine an optimal global path. However, an important point is that they do not hand a plan down to a lower level planner for execution, but rather maintain an internal representation that allows them to participate directly in the control of the vehicle based on its current state. $A^{*}$ yields a set of pointers within the map grid that point toward the goal, as depicted by the small arrows in Figure 6. During execution, this grid may be indexed by the current vehicle position to yield a path towards the goal which is optimal based on the information available in the map at that time.

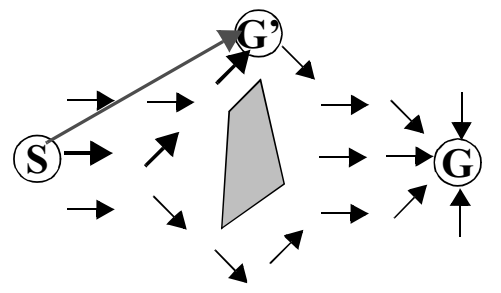

Figure 6: Following Gradient Field to determine intermediate goal heading
The Internalized Plans (Payton, 1990) approach uses a detailed map to perform an $\mathrm{A}^{*}$ search from the goal(s) back toward the start point to create a "Gradient Field" towards the goal. The type and slope of the terrain, among other factors, is used to estimate the cost of traversal between grid cells. During run-time, the grid cell containing the current vehicle location is identified, and the Gradient Field pointers are followed forward to the point $\mathbf{G}^{\prime}$ in Figure 6; the desired heading to reach the goal is that from the current location $\mathbf{S}$ to $\mathbf{G}^{\prime}$, and a series of votes with its peak at that value is sent to the turn arbiter.

The D* planner (Stentz, 1993) also creates a grid with "backpointers" that represent information on how best to reach the goal from any location in the map. The map may initially contain no information, but is created incrementally as new information becomes available during the execution of a mission, and the arc traversal costs and backpointers are updated to reflect this new knowledge. The resulting global plan is integrated into DAMN as a behavior by determining, for each possible turn command, the weight $w$ of reaching the goal from a point along that arc a fixed distance ahead (the squares designated collectively as $\mathbf{S}^{\prime}$ in Figure 7). If $w_{\text {max }}$ and $w_{\text {min }}$ are the maximum and minimum values of $w$, then the vote for each turn command is determined as: $\left(w_{\max }-w\right) /\left(w_{\max }-w_{\min }\right)$. In the case that a point $\mathrm{S}$ ' is not represented on the grid, or if the goal cannot be reached from it, then the vote for that arc is set to -1 .

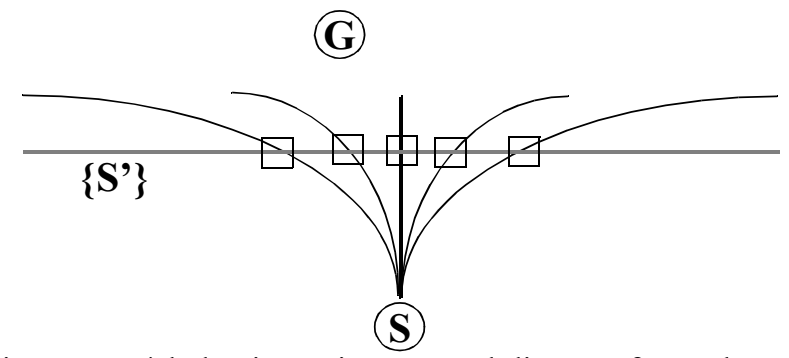

Figure 7: $\mathrm{D}^{*}$ behavior estimates goal distance for each arc

\section{Teleoperation}

Teleoperation is another possible mode in which a robotic system may need to operate. The STRIPE teleoperation system (Kay and Thorpe, 1993) provides a graphical user interface allowing a human operator to designate waypoints for the vehicle by selecting points on a video image and projecting them on to the surface on which the vehicle is travelling. STRIPE then fits a spline to these points and uses pure pursuit to track the path. When used in isolation, it simply sends a steering command to the controller; when used as a DAMN behavior, it sends a set of votes representing a Gaussian centered on the desired command. This allows the dynamic constraints and obstacle avoidance behaviors to be used in conjunction with STRIPE so that the safety of the vehicle is still assured. 


\section{Auxiliary Behaviors}

Various other auxiliary behaviors that do not achieve a particular task but issue votes for secondary considerations may also be run. These include the Drive Straight behavior, which simply favors going in whatever direction the vehicle is already heading at any given instant, in order to avoid sudden and unnecessary turns; and the Maintain Turn behavior, which votes against turning in directions opposite to the currently commanded turn, and which helps to avoid unnecessary oscillations in steering, the Follow Heading behavior which tries to keep the vehicle pointed in a constant direction, as well as various behaviors which allow user input to affect the choice of vehicle turn and speed commands.

\section{Combining Behavior Votes}

The voting strengths, or weights, of each behavior are specified by the user, and are then normalized by the arbiter so that their sum equals 1 . Because only the relative values are important, and because the magnitude of each behavior's votes vary according to their importance, DAMN is fairly insensitive to the values of these weights and the system performs well without a need to tweak these parameters. For example, the Obstacle Avoidance behavior has been run in conjunction with the Seek Goal behaviors with relative weights of 0.75 and 0.25 , respectively, and with weights of 0.9 and 0.1 , and in both cases has successfully reached goals while avoiding obstacles.

The vote weights of each behavior can also be modified by messages sent to the arbiter from a mode manager module. It can reconfigure the weights according to whatever top-down planning considerations it may have, and potentially could use bottom-up information about the effectiveness and relevance of a behavior (Payton et al., 1993). Different modes of operation that exclude some behaviors can be constructed by setting the weights those behaviors to 0 . A Mode Manager was developed at the Hughes Research Labs to be used with DAMN for this purpose, and at CMU Annotated Maps were integrated with DAMN to provide this capability (Thorpe et al, 1991).

As a simple example to illustrate the manner in which votes are issued and arbitrated within DAMN, consider the case in Figure 8 where two behaviors are active, one responsible for obstacle avoidance and the other for goal seeking (only five turn options are shown for simplicity). The magnitude of a vote is indicated by the size of a circle, with a large unfilled circle representing a vote of +1 , a large striped circle a value of -1 , and a small circle a value near 0 . Thus, the goal-seeking behavior is voting most strongly in favor proceeding straight and less favorably for a soft left turn, and voting against hard left or any right turns; the obstacle avoidance behavior is voting against a hard left or soft right, and allowing the other turns as acceptable, with soft left being the most favorable.

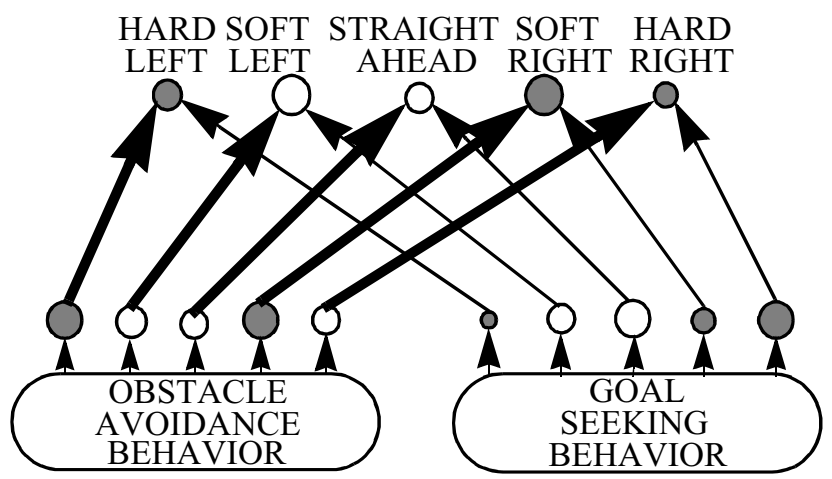

Figure 8: Command fusion in DAMN

Because avoiding obstacles is more important than taking the shortest path to the goal, the obstacle avoidance behavior is assigned a higher weight than the goal seeking behavior, as indicated by the thicker arrows in the diagram. The arbiter then computes a weighted sum of the votes it has received from each behavior, and the command choice with the highest value is selected and issued to the vehicle controller. In this case a soft left turn would be executed, since its weighted sum is the greatest, thus avoiding any obstacles while still more or less moving toward the goal. The favorableness of the selected turn command may also used to determine vehicle speed, so that, for example, the vehicle would slow down if a command is issued which will take the vehicle too far from the path to the goal point. Another possibility is to have a separate speed arbiter that would receive commands from behaviors that, given the current vehicle radius, determine the maximum speed that would satisfy their objectives.

\section{Experimental Results}

For the experiment described here, the system was configured with two behaviors, Obstacle Avoidance and Goal Seeking, which were described in the DAMN Behaviors section. The Turn Arbiter combined the votes from these two behaviors and issued a new steering command every $100 \mathrm{~ms}$. The goal points were on average 100 meters apart and the Goal Seeking behavior switched goals whenever it came within eight meters of the current target goal point. The weights were 0.8 and 0.2 for the Obstacle Avoidance and Goal Seeking behaviors, respectively. The obstacle weight was large relative to the goal weight to reflect the fact that avoiding obstacles is generally considered to be more important than approaching the goal at any given moment. The maximum vehicle speed was 2 meters/second in this experiment. A more detailed description of the experimental system can be found in Langer, Rosenblatt and Hebert (1994). 

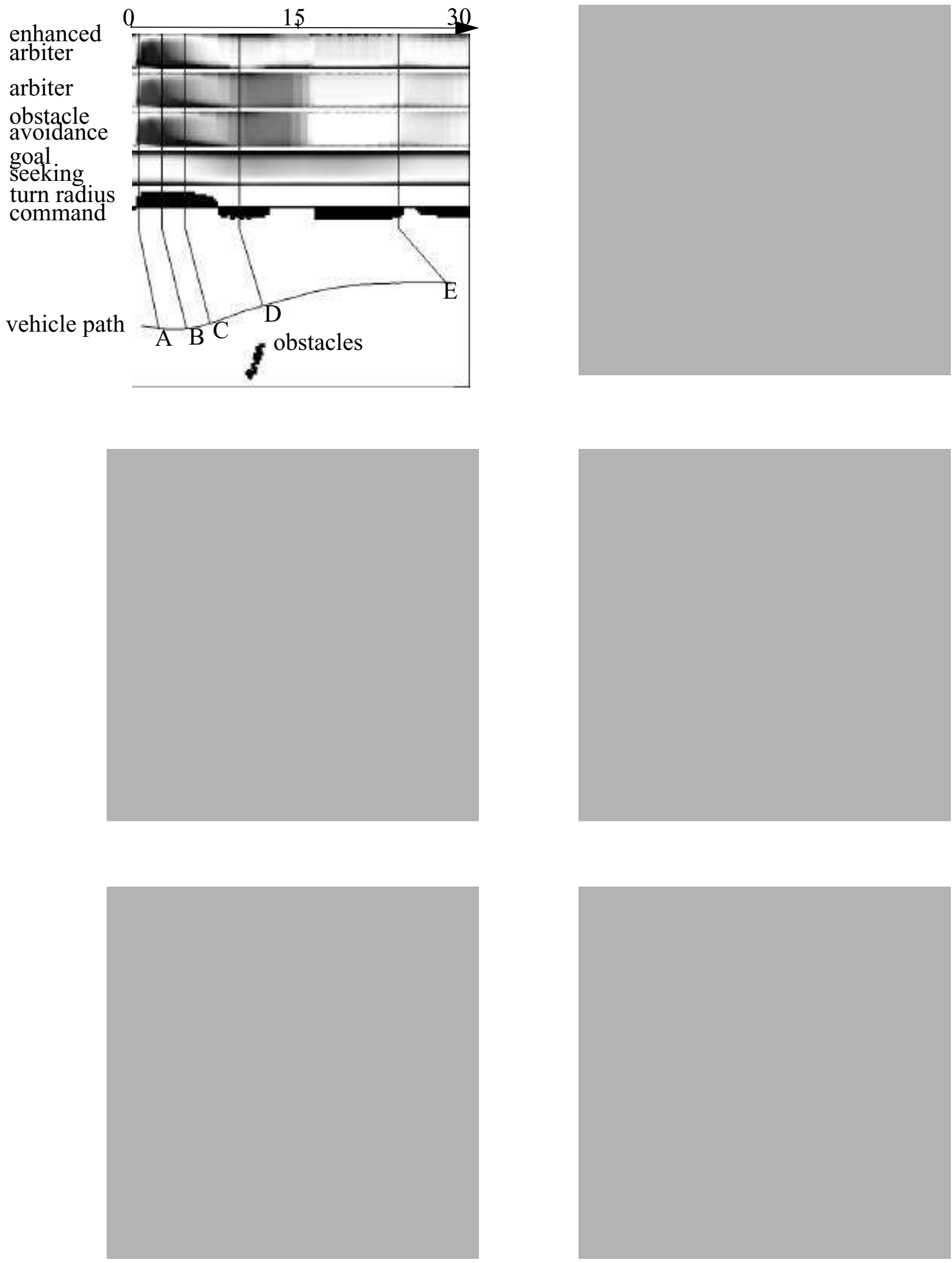

Figure 9: Vehicle path and corresponding votes over time, and vote distributions at five specified locations. 
Figure 9 illustrates the way the DAMN arbiter combined outputs from the behaviors. The first section of the figure shows the path of the vehicle as it travels around a set of obstacle points, and the distribution of votes for each of the behaviors and for the arbiter along that path. The horizontal axis is time, with the total length of the sequence being 30 seconds. For each module, the vertical axis is the set of turn choices from a hard left to a hard right turn. The votes were sampled every $200 \mathrm{~ms}$ for display purposes. The votes are displayed as shaded pixels, with the brightest pixels corresponding to the highest possible vote of 1.0 and the black pixels corresponding to the lowest possible vote of 1.0. In addition to the raw votes from the arbiter, the figure shows an enhanced display of the arbiter's distribution of votes, obtained by normalizing the pixel values using the actual minimum and maximum values at each time slot instead of the extreme possible values. The enhanced arbiter display is included to facilitate the visual interpretation of the vote distribution only and was not used for driving the vehicle. In addition to the vote distribution, Figure 9 also includes a graph of the commanded turn radius over time; the commanded turning radius is derived from the maximum of the vote distribution of the arbiter, as described in the Turn Arbiter section.

Five points of interest along the vehicle path, labeled as A through E, are shown in greater detail by the five graphs that follow in Figure 9. Each graph depicts the votes issued for each turn choice by the obstacle avoidance and goal behaviors, as well as the weighted sum of these votes as computed by the arbiter. The horizontal axis of each graph shows the possible turn radius choices encoded from -8 meters for hard left to +8 meters for hard right (curvature and turn radius are multiplicative inverses). The sequence of five points selected is typical of a path around a single obstacle.

At point $A$, the obstacle is first reported and the obstacle avoidance behavior generates high votes for turning left to go around the obstacle, and inhibits right turns with negative votes, as shown by the solid line in the graph. At the same time, the goal behavior's vote distribution is relatively flat around the straight direction since the vehicle is currently headed in the desired direction; this is shown by the dashed line. Because of the small relative weight of the goal behavior, the combined vote distribution in the arbiter, shown as a thicker solid line, is dominated by the votes received from the obstacle avoidance behavior; a left turn is therefore commanded. At point $B$, the obstacle is still close to the vehicle and the votes distributions are similar to the ones at $A$, thus maintaining the vehicle to the left of the obstacle.

At point $C$, the obstacle avoidance behavior is still voting in favor of a sharp left turn, but the votes for the softer left turns is now not as low as it was at $A$ or $B$, since the vehicle is now clearing the obstacles. At the same time, the goal behavior is starting to shift its votes towards turning right in order to bring the vehicle back to the target. The summed votes are still at a maximum for a left turn because of the greater weight of the obstacle avoidance behavior's votes, and so the arbiter continues to steer the vehicle well clear of the obstacles.

By the time the vehicle has reached point $D$, it is just passing the obstacles, so that the obstacle avoidance behavior is now only disallowing extreme right or left turns because of field of view constraints. The goal behavior is now able to have more influence over the chosen turn direction, and a right turn is now executed so that the desired vehicle heading is restored. Note that between points $C$ and $D$, there is a transition period where the turn radius chosen by the arbiter is neither the hard left favored by the obstacle avoidance behavior nor the medium right favored by the goal behavior. Instead, as turns other than hard left become acceptable to the obstacle avoidance behavior, and as the distribution of votes from the goal behavior shift further to the right, the vehicle is commanded to make ever softer left turns, and eventually turns to the right. As can be seen in the graph of the commanded turn radius in Figure 9, rather than abruptly switching modes from avoiding obstacles to following a heading, the transition proceeds smoothly and steadily as the situation gradually changes.

Finally, at point $E$, the vehicle has completely cleared the obstacles and they are no longer in the local map, so that the votes from the obstacle avoidance behavior are mostly +1 . The goal behavior now dominates completely and a right turn is commanded. Through the interaction of the avoid obstacles and goal behaviors, the vehicle was thus able to successfully circumvent an obstacle and regain its desired heading. This simple experiment shows that the arbiter combines the preferred commands from different behaviors in an efficient and natural way.

\section{Future Work}

DAMN has proven to be very effective as an architecture which greatly facilitates the integration of a wide variety of different vehicle navigation subsystems; however, as DAMN is used for increasingly complex tasks where many behaviors may be issuing votes concurrently, there will be a greater need to have the semantics of the voting process defined more carefully. By explicitly representing and reasoning about uncertainty within the decision-making processes, a system can be created whose effects are welldefined and well-behaved.

We have decided to pursue utility theory as a means of defining the semantics of the voting and arbitration processes. Because we are attempting to decide which among a set of possible actions to take, it is natural to make judgments on the usefulness of each action based on its consequences. If we assign a utility measure $U(c)$ for each possible consequence of an action, then the expected utility for an action $a$ is: 


$$
U(a)=\sum_{c} U(c) \cdot P(c \mid a, e)
$$

where $P(c \mid a, e)$ is the probability distribution of the consequence configuration $c$, conditioned upon selecting action $a$ and observing evidence $e$ (Pearl, 1988). Thus, if we can define these utilities and probabilities, we can then apply the Maximum Expected Utility (MEU) criterion to select the optimal action based on our current information.

If utility theory is to be applied to the problem of evidence combination and action selection for mobile navigation tasks, a means for defining the utility functions must be provided. Behaviors are defined in order to achieve some task, so it is fair to assume that there must be at least an implicit measure of "goodness" or utility with respect to that task. For example, an obstacle avoidance behavior's task is to maximize distance to obstacles, so that the distance from the vehicle to the nearest obstacle could be used as a measure of goodness. Likewise, proximity to the current goal could be used for the goal-based behaviors, as proximity to the center of a road (or lane) could be used by road following modules.

We will also be investigating command fusion using a local map for turn and speed arbitration, as was done for the field of regard arbiter. This will allow the arbiter to ensure consistency in the voting process by transforming votes as the vehicle moves. This will also allow behaviors to vote on where to travel rather than how to steer; however, complications due to the non-holonomic constraints of the vehicle will need to be addressed.

Another issue that needs to be addressed is the coupling between turn and speed commands. Currently, the turn and speed arbiters operate independently. Turn behaviors can use vehicle speed as one of their inputs and vice versa; however, there is currently no mechanism which allows behaviors to vote for a combination of turn and speed. Research is ongoing to determine a command representation which allows both coupled and independent voting, and is still efficient enough for real-time purposes.

\section{Conclusion}

DAMN is designed so that various behaviors can be easily added or removed from the system, depending on the current task at hand. Although the modules described in the DAMN Behaviors section all use very different paradigms and representations, it has been relatively straightforward to integrate each and every one of them into the framework of DAMN. Sensor fusion is not necessary since the command fusion process in DAMN preserves the information that is critical to decision-making, yielding the capability to concurrently satisfy multiple objectives without the need for centralized bottlenecks. All of the behaviors described have been used in conjunction with each other in various configurations, yielding systems that were more capable than they would have been otherwise. Conceptually, three levels of competence have been implemented in DAMN thus far, as shown in Figure 10. These levels of competence are convenient for describing the incremental manner in which the system's capabilities evolve; however, it is important to note that all behaviors co-exist at the same level of planning. The importance of a behavior's decisions is reflected by the weighting factor for its votes, and is in no way affected by the level of competence in which it is described.

\begin{tabular}{|c|c|c|}
\hline & & GOALS \\
\hline & ACTION & $\begin{array}{l}\text { Subgoals } \\
\text { Gradient Fields } \\
\mathrm{D}^{*}\end{array}$ \\
\hline SAFETY & $\begin{array}{r}\mathrm{Ro} \\
\mathrm{Cl} \\
\mathrm{T}\end{array}$ & $\begin{array}{l}\text { llowing } \\
\text { lountry } \\
\text { eration }\end{array}$ \\
\hline Vel & ynamics & \\
\hline Obs & $\begin{array}{l}\text { Avoidance } \\
\text { iliary }\end{array}$ & \\
\hline
\end{tabular}

Figure 10: Evolutionary system development in DAMN

The safety behaviors are used as a first level of competence upon which other levels can be added. The second level of competence, Action, has been implemented using road following, cross-country navigation, and teleoperation behaviors; they have all been run together with the obstacle avoidance behavior to provide various forms of generating purposeful action while maintaining safety (Thorpe et al, 1991). The third level of competence is comprised of the various map-based goal-seeking behaviors. Cross-country behaviors have been combined with goaloriented behaviors to produce directed off-road navigation (Keirsey, Payton and Rosenblatt, 1988; Stentz, 1993).

The scenario described in the Experimental Results section shows that a reactive architecture is capable of navigating cross-country terrain while pursuing mission objectives such as goal locations. The reactive nature of the system is possible because the perception module simply produces lists of binary obstacles as needed by the navigation system. If the output of the perception system were instead a detailed geometric model of the terrain, then planning would require significantly more computation, thus precluding the use of a reactive approach.

The current design of DAMN, with the arbiter taking inputs from a set of specialized behaviors, is flexible enough to allow for a variety of other behaviors to be combined or substituted. In addition to its use on the CMU Navlab vehicles, DAMN has also been used at Martin Marietta (now Lockheed Martin), Hughes Research Labs, and Georgia Institute of Technology. Like other behavior-based 
architectures, it avoids sensory and decision-making bottlenecks and is therefore able to respond in real-time to external events; however, it imposes no constraints on the nature of the information or the processing within a behavior, only on the interface between the behavior and the command arbiter. Furthermore, the behaviors are not subject to any timing constraints; each behavior operates asynchronously and in parallel.

Non-reactive behaviors may use plans to achieve goals and coordinate with other agents; thus, like centralized or hierarchical architectures, DAMN is able to assimilate and use high-level information. Finally, unlike architectures with prioritized modules, DAMN's vote arbitration scheme allows multiple goals and constraints to be fulfilled simultaneously, thus providing goal-oriented satisficing behavior without sacrificing real-time reactiveness.

\section{Acknowledgments}

The UGV project is supported by ARPA under contracts DACA76-89-C-0014 and DAAE07-90-C-R059, and by the National Science Foundation under NSF Contract BCS9120655. Julio Rosenblatt is supported by a Hughes Research Fellowship. The author would like to acknowledge the cooperation in vehicle experimentation of Martial Hebert and Dirk Langer, the support and guidance of Dave Payton and Chuck Thorpe, as well as the shared efforts and technical support of the Navlab group at Carnegie Mellon University.

\section{Appendix 1: Responses to issues raised}

1. Coordination-- How should the agent arbitrate/ coordinate/cooperate its behaviors and actions? Is there a need for central behavior coordination?

Centralized architectures provide the ability to coordinate, in a coherent fashion, multiple goals and constraints within a complex environment, while decentralized architectures offer the advantages of reactivity, flexibility, and robustness. The DAMN architecture takes the position that some centralization is needed, but the right level must be chosen so that it does not create a bottleneck, and the interfaces must be defined so as to avoid being overly restrictive.

Rather than imposing an hierarchical structure or using a prioritized behavior-based systems to effect a traded control system, the Distributed Architecture for Mobile Navigation takes a shared control approach where several modules concurrently have some responsibility for control of the robot. In order to achieve this, a common interface is established so that modules can communicate their intentions without regard for the level of planning involved.

Votes from all behaviors are used in determining what the next action should be, so that compromises are made when possible; however, if two behaviors suggest actions that can not be reconciled, then one of the two must be chosen. Metalevel control may be exerted so that behaviors with mutually exclusive goals do not operate simultaneously; however, plans are not used in a top-down fashion but rather as a source of advice, so that the flexibility of the reactive level is preserved.

The hypothesis proposed here is that centralized arbitration of votes from distributed, independent decisionmaking processes provides coherent, rational, goal-directed behavior while preserving real-time responsiveness to its immediate physical environment.

2. Interfaces-- How can human expertise be easily brought into an agent's decisions? Will the agent need to translate natural language internally before it can interact with the world?

Natural language is not needed for human-computer interaction, and in many domains would actually be more difficult for an operator to use than other modes of interaction. For example geometric information or mission goals can be more easily specified via a Graphical User Interface (GUI), and direct control of the robot can be better effected through the use of a joystick, for example.

In DAMN, the user may specify which behaviors are to be active and what their relative weights should be, either a priori or during run-time via a GUI, thus providing metalevel control. Behaviors that support teleoperation have also been implemented, so that the user may specify the robot path via waypoints in a video image, by using a joystick, or by typing simple keystroke commands. As with all other behaviors, the user's input is expressed as votes which are then combined with the votes of other behaviors and arbitrated.

3. Representation-- How much internal representation of knowledge and skills is needed? How should the agent organize and represent its internal knowledge and skills? Is more than one representational formalism needed?

The DAMN system only requires votes from each behavior, so that each module is free to use whatever representation and paradigm best serves for its particular task. However, the selection of which behaviors should be active at any given time is currently done in a fairly rudimentary way; an explicit representation of each behavior's skills would allow for more dynamic and flexible responses to unforeseen situations, and may also facilitate learning.

4. Structural-- How should the computational capabilities of an agent be divided, structured, and interconnected? How much does each level/component of an agent architecture have to know about the other levels/components?

The capabilities of an agent should be divided up as finely as is practical among task-achieving behaviors which operate asynchronously. They should be completely modular and independent, so that new capabilities may be added in an 
evolutionary fashion without a need to disrupt or modify existing functionality.

Ideally, higher level components that exert meta-level control should only need to know which skills are provided by the lower levels, without any knowledge of how those skills are implemented whatsoever.

5. Performance-- What types of performance goals and metrics can realistically be used for agents operating in dynamic, uncertain, and even actively hostile environments?

Objective quantitative evaluation of mobile robot systems has historically proven to be very difficult, and the task is doubly difficult when attempting to compare entire architectures because there are so many degrees of freedom in the design and implementation of a mobile robot system. The most straightforward means of evaluation is to attempt to define measures of the quality of the path which a vehicle has traversed under autonomous control. The measures I suggest within the body of the paper are the path's utility, smoothness, and accuracy:

Utility Measures One means of evaluating architectures is to use measures of "goodness" like those used to define the utility of each action for the various behaviors. Although evaluating the performance based on the utility measures defined in the Future Work section would appear to be circular, they are the best measures available. Furthermore, their evaluation is not vacuous given that uncertainty exists in all aspects of a system: in the declarative knowledge, sensed information, procedural knowledge, and in the effects of its actions and the actions of other agents; thus the expected utility will in general not agree with the actual utility that can be measured for those actions that are taken.

Let the goodness value for behavior $b$ at a state $s$ be computed by the function $g_{b}(s)$. Then one possible measure for the utility, with respect to behavior $b$, of consequence $c$ is the change in this measure of goodness with respect to the current state $s_{0}$ :

$$
U_{b}(c)=\Delta g_{b}=g_{b}(c)-g_{b}\left(s_{0}\right)
$$

However, the value of this measure can be arbitrarily large, so it must be normalized and assigned a consistent semantic meaning for all behaviors. One means of normalization would be to divide by the larger of the two goodness measures:

$$
U_{b}(c)=\Delta g_{b} / \operatorname{Max}\left(g_{b}(c), g_{b}\left(s_{0}\right)\right)
$$

This would bound the absolute value of $U_{b}(c)$ to be no greater than 1. It would also have the effect that, for the same $\Delta g_{b}$, the utility of an action would be greater when the goodness measure is small than when it is large. This appears to be desirable for behaviors such as obstacle avoidance whose importance should grow as the situation worsens; however, the suitability of this measure is less clear for other behaviors such as goal-seeking.

Another possibility for a normalized utility measure is analogous to the one used by the $\mathrm{D}^{*}$ behavior described in the Goal-Directed Behaviors section. After computing $g_{b}(c)$ for each possible consequence $c$, we determine the maximum and minimum values $g_{\max }$ and $g_{\min }$, and use the following measure:

$$
U_{b}(c)=\left(g_{b}(c)-g_{\text {min }}\right)^{\prime}\left(g_{\text {max }}-g_{\text {min }}\right)
$$

While this measure has the desirable property of being bounded by the interval $[0,1]$, it has the potentially undesirable property that the range of utility values will always completely span that interval. Thus, for example, if all potential actions have exactly the same utility except for one that has a slightly higher value, then the normalized utility values will be 1 for that one action and 0 for all the rest.

Path Smoothness Another general measure of the quality of the path is the smoothness with which it is controlled. Integrating the squared derivative of curvature along the vehicle's actual path provides a measure of smoothness that can be applied to the architecture (Kamada, Naoi and Goto, 1990), and the derivative of acceleration, or jerk, provides a measure of smoothness in the vehicle's speed as well as steering. Integrating the squared vehicle curvature along the vehicle's path may also be useful as a measure of smoothness, which reflects consistency in decision-making and the ability to anticipate events.

Path Accuracy The accuracy of the path, i.e. the extent to which the commanded path matches the actual path taken by the vehicle, can also provide an important means of evaluating an architecture and its planning subsystems. Path accuracy can be measured by integrating the squared error between estimated and actual vehicle poses. If the system commands trajectories which are not physically realizable, the actual path taken may deviate significantly. Likewise, if large latencies exist in the system and are not adequately compensated for, the commanded path will only begin to be executed well after the system intended it.

6. Psychology-- Why should we build agents that mimic anthropomorphic functionalities? How far can/should we draw metaphoric similarities to human/animal psychology? How much should memory organization depend on human/ animal psychology?

If the goal of the system is to produce an efficient means of controlling artificially constructed agents, as is the case with DAMN, then existing systems, i.e. humans and other animals, should serve merely as an inspiration, never as a constraint. Furthermore, good software engineering practices dictates that robotic systems must evolve in a more orderly fashion than their biological counterparts. 
7. Simulation-- What, if any, role can advanced simulation technology play in developing and verifying modules and/or systems? Can we have standard virtual components/test environments that everybody trusts and can play a role in comparing systems to each other? How far can development of modules profitably proceed before they should be grounded in a working system?

Simulation technology can be a very important tool for developing systems which are eventually realized in a physical implementation, but the former must not replace the latter. In my experiences in developing navigation systems, simulation has often played a key role in their success. Simulation provides a means to develop and extensively test the system with minimal resources and without risking physical damage to a robot or its surroundings. Subsequent testing on a real robot then provides not only a means of validating the system, but also a means of discovering the flaws in the simulation so that its fidelity may be improved for future use.

Any attempt to standardize the virtual components of a robotic system would be premature at this point, as there is still much debate as to what form the decomposition of the architecture should take. Also, different needs in terms of complexity, responsiveness, and optimality will require different architectures which make different trade-offs.

Standardized test environments, however, are something that is sorely needed so that architectures may be compared to each other in a systematic way. Again, different environments with different demands in complexity, responsiveness, and optimality will be needed to highlight the relative strengths and weaknesses of each architecture in various domains. While a real robot provides the best testbed, simulation can also play an important role because of its accessibility, its low cost, and because it provides the ability to record every aspect of a trial for analysis and for reproducing results.

8. Learning-- How can a given architecture support learning? How can knowledge and skills be moved between different layers of an agent architecture?

For the sake of generality, the higher levels of a robot architecture tend to use knowledge-based systems containing explicit representations; thus, given a sufficient domain model, these levels may be amenable to techniques such as explanation-based learning. For the sake of efficiency, the lower levels of an architecture tend to use compiled routines and implicit representations, and therefore the various forms of learning via gradient descent are more applicable. Knowledge learned at the higher levels may be compiled via mechanisms such as chunking, and eventually migrated to the lower levels for repeated use.
There currently is no learning within the DAMN architecture. The most important set of parameters in DAMN is the behavior weights. While setting these weights for a small number of behaviors has been simple, doing so for larger systems with more complex interactions could be problematic. Reinforcement learning would seem to be a natural means of acquiring information regarding the utility of various actions in different circumstances, and the relevance and usefulness of each behavior in particular situations might also be learned through techniques such as genetic algorithms. The mode manager changes behavior weights according to a precomputed schedule, but adaptive techniques such as those in (Maes, 1991) are necessary for dealing with a wider variety of situations and for fully autonomous control.

\section{References}

Albus, J., McCain, H. and Lumia, R. (1987) NASA/NBS Standard Reference Model for Telerobot Control System Architecture (NASREM), NBS Tech. Note 1235, Gaithersburg, MD.

Arkin, R. (1987) Motor Schema Based Navigation for a Mobile Robot: An Approach to Programming by Behavior. Proceedings of the International Conference on Robotics and Automation, Atlanta, GA, 1993.

Brooks, R. (1986) A Robust Layered Control System for a Mobile Robot. IEEE Journal of Robotics and Automation vol. RA-2, no. 1, pp. 14-23, April 1986.

Brooks, R. (1993) Intelligence Without Reason. Proceedings of the International Joint Conference on Artificial Intelligence, Chambery, France, August 1993.

Daily, M., Harris, J., Keirsey, D., Olin, K., Payton, D., Reiser, K., Rosenblatt, J., Tseng, D. and Wong, V. (1988) Autonomous Cross-Country Navigation with the ALV. IEEE Conference on Robotics and Automation, Philadelphia, PA, April, 1988; (also appears in DARPA Knowledge Based Planning Workshop, December, 1987 pp 20-1 to 20-10).

Kamada, H., Naoi, S. and Goto, T. (1990) A Compact Navigation System Using Image Processing and Fuzzy Control. Proceedings of IEEE Southeastcon, New Orleans, April 1-4, 1990

Kay, J., Thorpe, C. (1993) STRIPE Supervised Telerobotics Using Incremental Polygonal Earth Geometry. Proceedings of Intelligent Autonomous Systems Conference, Pittsburgh, PA, February 15-18, 1993, IOS Press. 
Keirsey, D.M., Payton, D.W. and Rosenblatt, J.K. (1988) Autonomous Navigation in Cross-Country Terrain. Proceedings of Image Understanding Workshop, Cambridge, MA, April, 1988.

Langer, D., Rosenblatt, J. and. Hebert, M. (1994) A Behavior-Based System For Off-Road Navigation. IEEE Journal of Robotics and Automation, vol. 10, no. 6, pp. 776-782, December 1994; (also appears as An Integrated System For Autonomous Off-Road Navigation. Proceedings of the IEEE International Conference on Robotics and Automation, San Diego, May 1994).

Lee, C. (1990) Fuzzy Logic in Control Systems: Fuzzy Logic Controller -- Parts I and II. IEEE Transactions on Systems, Man and Cybernetics, Vol 20 No 2, March/April 1990.

Maes, P. (1991) A Bottom-Up Mechanism for Action Selection in an Artificial Creature. Adaptive Behavior: From Animals to Animats, edited by S. Wilson and J-A Meijer, MIT Press.

Nilsson, N. (1980) Principles of Artificial Intelligence. Tioga Pub. Co., Palo Alto, Calif.

Nilsson, N. (1984) Shakey the Robot, SRI Tech. Note 323, Menlo Park, Calif.

Payton, D. (1986) An Architecture for Reflexive Autonomous Vehicle Control. IEEE International Conference on Robotics and Automation, San Francisco, CA, April 7-10, 1986, pp. 1838-1845.

Payton, D. (1990) Internalized Plans: A Representation for Action Resources, Robotics and Autonomous Systems, 6(1), 1990, pp. 89-103. (Also in Designing Autonomous Agents, ed. Pattie Maes, MIT Press, Cambridge, Mass. 1991, pp. 89-103.)

Payton, D., Rosenblatt, J. and Keirsey, D. (1990) Plan Guided Reaction. IEEE Transactions on Systems Man and Cybernetics, 20(6), pp. 1370-1382.

Payton, D., Keirsey, D., Kimble, D., Krozel, J. and Rosenblatt, J. (1993) Do Whatever Works: A Robust Approach to Fault-Tolerant Autonomous Control. Journal of Applied Intelligence, Volume 3, pp 226-250.

Pearl, J. (1988) Probabilistic Reasoning in Intelligent Systems: Networks of Plausible Inference. Morgan Kaufmann Publishers.

Pomerleau, D. (1992) Neural Network Perception for Mobile Robot Guidance, Ph.D. dissertation, Carnegie-Mellon Technical Report CMU-CS-92-115, Pittsburgh, PA.
Rosenblatt, J. (1995) DAMN: A Distributed Architecture for Mobile Navigation. Proceedings of AAAI Spring Symposium on Lessons Learned from Implemented Software Architectures for Physical Agents, H. Hexmoor and D. Kortenkamp (Eds.), Menlo Park, CA: AAAI Press.

Rosenblatt, J. and Payton, D. (1989) A Fine-Grained Alternative to the Subsumption Architecture for Mobile Robot Control. Proceedings of the IEEE/INNS International Joint Conference on Neural Networks, Washington DC, vol. 2, pp. 317-324, June 1989; (also appears in 1989 AAAI Spring Symposium Series).

Rosenblatt, J. and Thorpe, C. (1995) Combining Multiple Goals in a Behavior-Based Architecture. Proceedings of International Conference on Intelligent Robots and Systems (IROS), Pittsburgh, PA, August 7-9, 1995.

Rosenschein, S. and Kaelbling, L. (1986) The Synthesis of Digital Machines with Provable Epistemic Properties. Proceedings of Theoretical Aspects of Reasoning about Knowledge. pp 83-98.

Simmons, R., Lin, L.J., Fedor, C. (1990) Autonomous Task Control for Mobile Robots. Proceedings of IEEE Symposium on Intelligent Control, Philadelphia, PA, September 1990.

Stentz, A. (1993) Optimal and Efficient Path Planning for Unknown and Dynamic Environments. Carnegie-Mellon Technical Report CMU-RI-TR-93-20, Pittsburgh, PA.

Stentz, A. and Hebert, M. (1994) A Complete Navigation System for Goal Acquisition in Unknown Environments. Carnegie-Mellon Technical Report CMU-RI-TR-94-7, Pittsburgh, PA.

Thorpe, C., Amidi, O., Gowdy, J., Hebert, M. and Pomerleau, D. (1991) Integrating Position Measurement and Image Understanding for Autonomous Vehicle Navigation. Proceedings of Workshop on High Precision Navigation, Springer-Verlag Publisher.

Yen, J. and Pfluger, N. (1992) A Fuzzy Logic Based Robot Navigation System. Proceedings of AAAI Fall Symposium on Intelligent Applications of Artificial Intelligence to Real-World Autonomous Mobile Robots, Boston, MA, 1992.

Zadeh, L. (1973) Outline of a New Approach to the Analysis of Complex Systems and Decision Processes. IEEE Transactions on Systems, Man and Cybernetics, Vol 3 No 1, January 1973. 